\title{
REFERENCE
}

1. H. Rossi, Vector fields on analytic spaces, Ann. of Math. 78 (1963), 455-467.

Princeton University

\section{ON PIECEWISE LINEAR IMMERSIONS}

\section{MORRIS W. HIRSCH}

The purpose of this note is to prove an existence theorem for immersions of piecewise linear manifolds in Euclidean space. A more comprehensive theory of piecewise linear immersions has been worked out by Haefliger and Poenaru [1].

All maps, manifolds, microbundles, etc. are piecewise linear unless the contrary is explicitly indicated.

Let $M$ be a manifold without boundary, of dimension $n$. Denote the tangent microbundle of $M$ by $\tau_{M}$, and the trivial microbundle over $M$ of (fibre) dimension $k$ by $\epsilon^{k}$. Let

$$
\nu: M \stackrel{i}{\rightarrow} E \stackrel{j}{\rightarrow} M
$$

be a microbundle of dimension $k$ such that $E$ is a manifold. An immersion of $M$ in $R^{n+k}$ is a locally one-one map $f: M \rightarrow R^{n+k}$.

I say $f$ has a normal bundle of type $\nu$ if there is an immersion $g: E \rightarrow R^{n+k}$ such that $g i=f$. (It is unknown whether $f$ necessarily has a normal bundle, or whether all normal bundles of $f$ are of the same type.)

The converse of the following theorem is trivial.

Theorem. Assume that if $k=0$, then $M$ has no compact component. There exists an immersion of $M$ in $R^{n+k}$ having a normal bundle of type $\nu$ if there exists an isomorphism

$$
\phi: \tau_{m} \oplus \nu \rightarrow \epsilon^{n+k}
$$

Proof. We may assume that $i(M)$ is a deformation retract of the total space $E$ of $\nu$. By Milnor [3], $\tau_{E} \mid i(M)$ is isomorphic to $\tau_{M} \oplus \nu$; it follows from the existence of $\phi$ that $\tau_{E}$ is trivial. According to [3]

Received by the editors July 29, 1964. 
there is a parallelizable differential structure $\alpha$ on $E$ compatible with the piecewise linear structure. Let $h: E_{\alpha} \rightarrow R^{n+k}$ be a differentiable immersion, which exists by Hirsch [2] or Poenaru [4]. (If $k=0$, the assumption that $M$ has no compact component is used here.) Approximate $h$ by a piecewise linear immersion $g: E \rightarrow R^{n+k}$, using the theory of $C^{1}$ complexes of Whitehead [5]. Clearly $g i: M \rightarrow R^{n+k}$ is an immersion having a normal bundle of type $\nu$.

REMARKs. (1) The assumption that $M$ is unbounded is unnecessary, since a bounded manifold can be embedded in its interior. However, $\tau_{M}$ must be redefined if $M$ has a boundary.

(2) It is not hard to define the concepts of "immersion plus normal bundle"-essentially an immersion of $E$-and of a "regular homotopy" of these; one can then prove a uniqueness theorem.

\section{BibLIOGRAPHY}

1. A. Haefliger and V. Poenaru, La classification des immersions combinatoires, Publ. Math. I.H.E.S., no. 23, pp. 75-91.

2. M. W. Hirsch, On imbedding differentiable manifolds in euclidean space, Ann. of Math. 73 (1961), 566-571.

3. J. Milnor, Microbundles and differentiable structures, (mimeographed). Princeton Univ., Princeton, N. J., 1961.

4. V. Poenaru, Sur la theorie d'immersions, Topology 1 (1962), 81-100.

5. J. H. C. Whitehead, On $C^{1}$ complexes, Ann. of Math. 41 (1940), 809-824.

UNIVERSITY OF CAMBRIDGe, ENGLAND 